\title{
1 Mapping the Alaskan Moho
}

2 Meghan S. Miller ${ }^{1}$ and Louis Moresi ${ }^{2}$

3

$4{ }^{1}$ Australian National University, Research School of Earth Sciences, Canberra, ACT 2601

52 University of Melbourne, School of Earth Sciences, Parkville, VIC 3010

7 Abstract

9 We present a series of Moho depth maps for the Alaskan region based upon $\mathrm{P}$ receiver function

10 estimates using data from all available broadband instrumentation from 1999 to April 2018 11 including the USArray Transportable Array. The average Moho depth beneath individual 12 broadband stations are presented first as spot measurements and then used to produce a series

13 of interpolated smooth surfaces by an adaptive triangulation process followed by the fitting of a 14 bi-cubic spline. The interpolated surfaces include a measure of confidence in the interpolation 15 and can be used to assess and determine a preferred model. The resulting Moho depth map 16 (single continuous surface) provides a reasonable estimate of the Earth's outermost layer 17 thickness beneath Alaska as constrained by receiver functions, for use in applications such as 18 tomography, regional-scale interpretations, or simulations of seismic waves. The models are 19 provided as a python module with examples in the form of jupyter notebooks. Our original 20 workflow is provided to allow updates to this dataset or use with other similar datasets.

Introduction

24 The Alaskan crust is composed of a mosaic of oceanic and continental terranes that range in age 25 from Proterozoic to Cenozoic (Plafker and Berg, 1994). These terranes have been deformed and 
displaced through geologic time, yet their structure reflects both their origins and the

27 heterogeneous distribution of strain. Due the remote and harsh conditions in much of the region

28 there has been very sparse broadband seismic data coverage outside of south-central Alaska 29 until recently.

In September 2017 the final USArray Transportable Array (network code TA) seismic station was installed in Alaska. This was the last of 280 instruments deployed as a grid across Alaska and

33 northwest Canada as part of the EarthScope program which was proposed nearly 20 years ago

34 (Meltzer et al., 1999; Rosen 2017). This is the final stage of the continent-wide experiment that

35 has been ongoing since 2004. These instruments complement and greatly expand the number

36 and distribution of seismic stations that have previously been, or are permanently operating. The

37 data from all these seismic stations allows construction of a map of the average crustal architecture, along with investigation into deeper structure and a more comprehensive seismicity catalog for the surrounding region.

41 With the current distribution of broadband instruments across Alaska there is an opportunity to 42 make a regional map of Moho (Mohorovičić discontinuity) depth, assumed to coincide with the 43 crust-mantle boundary, or Moho. Although locally the Moho can be incredibly complex and have 44 variations in depth and magnitude of the velocity contrast across the boundary, an estimate of the 45 crust-mantle boundary structure is very important for geological and geophysical research. 46 Crustal models such as CRUST5.1 (Mooney et al., 1998) or CRUST2.0 (Bassin et al., 2000), and 47 more recently ones such as those based on EarthScope data (Gilbert, 2012; Levander and Miller, 48 2012; Tape et al., 2012) and the EARS (EarthScope Automated Receiver Survey) project 49 (Crotwell and Owens, 2005; IRIS DMC, 2010) have been very valuable for the solid Earth 50 community. Here we used all available data (1999-2018) from broadband instruments to compute 51 P receiver functions, then determined the Moho depth at each station from the stacked receiver 
52 functions in order to make a series of maps of the Moho depth including a smoothed surface. We

53 note that although there are some areas across the state that still have limited data, others that

54 are very densely instrumented, and in addition areas of very complex crustal structure. This map,

55 plus the associated measures of confidence may be useful by the community until there are future

56 improvements on this model.

57

\section{$58 \quad$ Instrument Deployment}

60 Five hundred and five broadband seismic stations have been deployed across Alaska and Yukon

61 Territory (Figure 1), which have open access data that can be obtained from the IRIS Data

62 Management Center (DMC) at www.iris.edu (last accessed April 2018). The seismic stations are

63 from multiple networks, both permanent and temporary (see electronic supplementary Table S1

64 for DOI numbers), with the bulk of these from the EarthScope Transportable Array (network code

65 TA) and the Alaska Regional Network (network code AK).

67 Overall Data Quality and Availability

69 A range of broadband seismic data from teleseismic earthquakes that occurred between 1999

70 through April 2018 (inset Figure 1) were selected and processed (see Data and Resources for

71 more details). These waveform data were obtained within the FuncLab software (Eagar and

72 Fouch, 2012; Porritt and Miller, 2018) via the irisFetch.m MATLAB script (Trabant et al., 2012).

73 The data were chosen for events with magnitudes >M6.0 at epicentral distances between 30-98.3

74 degrees. This resulted in a total of 2200 earthquakes (Supplementary Table S2) and 505 seismic

75 stations (Supplementary Table S3), which yielded 136,838 preliminary P receiver functions

76 (PRFs) calculated in FuncLab. The PRFs were calculated using iterative time-domain 
77 deconvolution (Ligorria and Ammon, 1999) of the vertical component from the radial and

78 transverse components using a $1 \mathrm{~Hz}$ central frequency. These were manually edited with the

79 FuncLab trace editor based on high signal-to-noise direct arrivals and clean traces, resulting in a

80 final total of 36,370 PRFs. A subset of these results and the methodology used in the processing

81 are discussed in Miller et al. (2018). These receiver functions are based upon detailed, manual

82 inspection and selection of all the waveforms and resulting receiver function, which is unlike the

83 fully automated data product, EARS (Crotwell and Owens, 2005) available at

\section{4 https://doi.org/10.17611/DP/EARS.1.}

86 The individual receiver functions are stacked at each station using a simple summation to improve

87 the signal-to-noise ratio and emphasize coherent conversions (examples shown in Figure S1,

88 available in the electronic supplement to this article). This stacking can eliminate more complex

89 or subtle signals from anisotropy or dipping structures, but does provide an effective way to

90 enhance the primary signals from sub-horizontal velocity discontinuities like the Moho. These

91 gathers are converted from time to depth using the ak1351D velocity model (Kennett et al., 1995)

92 to provide an estimate of the average structure at depth beneath each station. To provide a broad

93 scale Moho depth map across the region, the Moho conversions were hand-picked for each of

94 the receiver function stacks within FuncLab (Porritt and Miller, 2018). These picks are used as

95 spot measurements of the average Moho depth at each seismic station to create a simple map

96 as shown in Figure 2 and are provided in Table S2, available in the electronic supplement to this

97 article.

98

\section{Observations}

100

101 In September 2017 the Transportable Array was fully installed across Alaska and into the 102 bordering regions of Canada (Rosen, 2017) and this provides the first region/state-wide glimpse 
of the crustal thickness. Although there are still some areas of limited data and some areas of very complex signals in the receiver functions, the Moho depth map (Figure 2) presents a new overview across this vast area. There is a primary trend, despite the large area, that the crustal thickness roughly correlates with topography. The high topography in the Alaska, St. Elias and 107 Brooks Ranges and even the Chugach Mountains, for example, have deep Mohos (Figure 2). 108 There are also abrupt changes in Moho depth across terrane boundaries and faults, such as the 109 Denali fault (DF) system as documented previously (e.g. Veenstra et al., 2006; Brennan et al., 110 2011; Allam et al., 2017 Miller et al., 2018), but also at across other terranes boundaries. An 111 example of these terrane boundaries is between the Yukon-Tanana (YTT) and Wrangellia 112 composite terranes (WCT) which has previously been under sampled by seismic data (Figure 113 2B). There are other indications that the inferred terranes and litho-boundaries may also have 114 distinct structural changes across them as seen in the litho-type simplified map in Figure 2B 115 (Wilson et al., 2015; Miller et al., 2018).

117 The receiver functions typically have a very clear, simple signal of the Moho as shown in Figure 118 S1A for station TA.H20K in north-central Alaska. However, within the accretionary complex 119 (purple in Figure 2B), the majority of the Moho picks are $>35 \mathrm{~km}$ and can reach down to nearly $12060 \mathrm{~km}$ (Table S2, available in the electronic supplement to this article). However, the crustal 121 structure is not as simple as the map of local averaged measurements portrays. The individual 122 receiver functions are remarkably complex, which is likely due to the collision and subsequent 123 subduction of the Yakutat terrane and the downgoing Pacific plate. At some stations there are 124 "two Mohos" and at others there is no shallow (overriding) plate conversion in the signal (Figures 125 S1B - AK.VRDI and S1C - AK.BMR). This is particularly evident along the southern margin of 126 Alaska when the Pacific plate is subducting and the Yakutat terrane is colliding and being 127 subducted. There are large differences in the Moho depth estimates at closely spaced stations. 128 This is likely to be due to not being able to image one Moho or one discontinuity within the crust. 
129 This is discussed in Miller et al. (2018), which presents the $\mathrm{P}$ receiver functions in more detail. It

130 is essential to be cautious and understand the geology and tectonics, and the methodology used

131 in the seismic imaging, when using the Moho picks from stacked receiver functions and not solely

132 use the Moho map to understand complex tectonics.

\section{Results}

136 The Moho depth associated with individual stations is a useful first step at interpreting the crustal

137 architecture. However, an interpolated surface of the Moho within the survey area, along with a 138 measure of the confidence in such an interpolation is important to understand the extent to which

139 Moho values can be interpolated away from the measurement location, which is important for 140 interpretations. The Moho surface can be used as a starting model for other seismological and 141 geophysical methods used to infer Earth structure.

\section{Smooth, interpolated model}

143 A piecewise linear interpolant can be constructed from a Delaunay triangulation of the data points 144 (Figure 2 and Supplementary Table S2, available in the electronic supplement to this article) but 145 the irregular spacing of the instruments produce artefacts in interpolation that suggest it may be 146 more appropriate to apply some smoothing. The STRIPACK and SSRFPACK routines (Renka $1471997 a, b)$ are used to construct triangulations and spline interpolants for the data. However, the 148 spacing is irregular enough that the fitting procedure employed by SSRFPACK does not converge 149 for any value of the smoothing parameters. Instead we build a triangulation using a smaller 150 number of nodal points with an interpolating spline that we can use as a model to be fitted to the 151 data. 
153 To build an interpolating surface, we first find a set of mesh points that reasonably represents the

154 distribution of the data while also being sufficiently regular that it is possible to construct the spline

155 surface accurately. A simple approach to finding a set of $p_{1}$ nodal points to represent the

156 instrument locations is to use a clustering algorithm on the coordinate vectors. We used the k-

157 means clustering from scipy (Jones et al, 2001) with random initial centroids to provide sets of

158 nodes for triangulation. The random nature of the mesh construction means that we cannot 159 determine the influence of $p_{1}$ from a single realisation of the mesh and instead we need to average

160 over a large number of meshes. A second parameter, $p_{2}$, for the model is associated with the 161 degree of tensioning of the interpolating splines. A value of zero corresponds to bilinear 162 interpolation, larger values produce increasingly smooth results with a scale that depends on the 163 number of sample points and the statistics of the noise in the data. As the latter is not well known 164 in this case, we view $p_{2}$ as a free parameter to be determined.

Fitting the model 1: confidence estimates

166 We make a first pass through the data to identify the extent to which individual data points can be 167 fitted by our choice of a single-valued, continuous interpolating surface. We systematically 168 exclude each point from the data set and use the other data points to predict its value for 20 169 different realizations of the triangulations for all values of the model parameters $\left(p_{1}, p_{2}\right)$. Each 170 time, we identify the containing triangle of every observation and attribute a distance weighted 171 average contribution to each node of this triangle. The predictions of the model for each data point 172 can be evaluated by computing the mean (Figure 3A) and r.m.s variation (Figure 3B) of the values.

174 We then attribute a score (Figure 3C) to each of the data locations based on their predictability: 175 points where the mean error and variance are both small receive a score of 1 - these points are 176 reliable but may not be good at discriminating between models. Points where small errors are 177 possible with some combination of parameters are accorded an additional score of 1 . Nodes 
178 where models are consistently unreliable are accorded a score of 0.1 . The scores for each 179 observation location are mapped to grid points by distance-weighted averaging and used to 180 provide the node-by-node confidence estimate required by SSRFPACK.

182 The complexity of signals in the receiver functions and therefore the simple, average estimate of 183 Moho depth beneath each station can lead to high variance between the different model 184 realizations (Figure 3B). There are a number of points where no smooth, continuous model can 185 fit the observation. This is likely due to the receiver functions ability to image multiple interfaces, 186 not just the Moho, and the variable magnitude of the signal from these velocity contrasts at 187 variable depths. These complexities make it difficult to pick the true Moho if there is a clear 188 velocity contrast from this discontinuity. Therefore, our estimate of error is highest in regions 189 where the Moho picks (Figure 2) have large differences between closely spaced stations. This is 190 particularly evident in southern Alaska where collision and subsequent subduction of the Yakutat 191 terrane and the down-going Pacific plate results in complex crustal (and lithospheric) structure. 192 In these regions in particular, the structure might better be represented as a multi-valued surface, 193 as we are likely to be imaging multiple layers, but, in this preliminary analysis, we simply report 194 these as points where the score for the chosen model parameterization is low.

196 We construct 250 triangulations for each value in the range of $p_{1}$. For every triangulation, we 197 partition the data into two: one set of points $(70-95 \%$, chosen at random) is used to fit the data for 198 each of values chosen for the smoothing parameter, $p_{2}$ and the remaining points used to evaluate 199 the prediction error of the resulting interpolants. A small number of points usually remain 200 unconstrained by our data (as we eliminate several points to evaluate the fit of the model). We 201 set the Moho depth at those points using the global litho 1.0 dataset (Pasyanos et al, 2014). In 202 addition, these points receive a very low weighting in the surface fitting algorithm (Renka, 1997b) 203 so that they do not strongly influence the fit to nearby points. The results are processed to 
204 determine the fit to the test data for each pair of $p_{1}, p_{2}$ using the node-by-node confidence score 205 in Figure 3C to weight the depths in the spline fit.

207 The process of fitting the model is summarized in Figure 4 using two alternative measures of fit 208 over all the different grids and partitioning of the data. In one case (Figure 4A) we show the fit 209 only to the test data sets, and in the second case (Figure 4B), we form a combined error which is 210 the misfit for all the data, including the training data. Around the best fitting points in each case 211 are a number of models with a misfit within $1.25 \%$ of the minimum value. The models based only 212 on fitting the test data are the smoothest models that satisfy the observations whereas those that 213 minimize the combined error are more likely to over-fit the data. There are several models which 214 are within $1 \%$ to $2 \%$ of the minimum of each of the measures and these appear to represent a 215 good balance between overfitting and over-simplifying the model. Our preferred model is an 216 average of these cases; we also include the two end-member best-fit models for the purposes of 217 comparison.

218 Model: data files and software

219 The models are provided in a python package miller_alaskamoho_srl2018 220 (https://pypi.org/project/miller_alaskamoho_srl2018/) that can be installed 221 through the pip python package management system. The package includes documentation in 222 the form of example jupyter notebooks. Installation instructions are available in the electronic 223 supplement to this article.

225 The model itself takes the form of 1) a numpy record array containing the raw Moho data plus the 226 model statistics reported above and the model scoring used to perform the surface fitting, and 2) 227 a numpy array of longitude, latitude, depth for the Moho surface and Moho slope $(s=|\nabla h|$ where $228 h$ is the depth of the Moho surface) computed on a dense, regular triangulation. The models are 
accessed through the package as instances of a class that includes the relevant triangulation and

230 interpolation methods (see electronic supplement for details).

232 We supply three alternative versions of this surface as discussed above: 1) our preferred 233 ensemble average model constructed from all models that lie within $1.25 \%$ of each of the end

234 member cases as shown in Figure 5A, 2) the smooth end-member that minimizes the misfit of the 235 test data alone, and 3), the end member that minimizes the misfit of all the data: test and training 236 sets combined.

238 We also provide the source code in the form of jupyter notebooks to process the raw data, to 239 evaluate the model errors and to compute and interpolate the fine grid surfaces and their 240 gradients. These notebooks also allow the evaluation of other ensembles of models. We supply 241 example python code to read the data files, convert to various formats, and interpolate the surface 242 for plotting (instructions for accessing the models and software are available in the electronic 243 supplement to this article).

\section{Summary}

246 Our maps of the Moho beneath Alaska and the surrounding regions are based upon hand picks 247 from $\mathrm{P}$ receiver function station stacks calculated with Funclab (Eagar and Fouch, 2012; Porritt 248 and Miller, 2018) for all available stations between 1999 and April 2018. The receiver function 249 stacks provide an average Moho depth based upon a simple 1D velocity model conversion. 250 These depths are then used to produce an interpolated surface to predict the Moho across the 251 region, and to provide a measure of the confidence in such a map. Future improvements will 252 result for additional data collected by the TA and other permanent stations. This new data and 253 constraints, or other data products such as EARS (IRIS DMC, 2010), can be easily incorporated 
254 into the map and into the jupyter notebook scripts that generate the Moho surface and the 255 associated measures of confidence.

\section{Data and Resources}

257 The broadband seismic data used to produce the $\mathrm{P}$ receiver functions and resulting maps are 258 available from the IRIS Data Management Center at www.iris.edu (last accessed April 2018). The 259 network DOI codes are found in Supplementary Table 1, available in the electronic supplement 260 to this article. The receiver functions and the Moho picks were calculated and edited in FuncLab 261 1.8.1 (Porritt and Miller, 2018), which is available online at 262 https://robporritt.wordpress.com/software/. The figures were made with Generic Mapping Tools 263 (GMT; Wessel and Smith, 1991). We made extensive use of the following python packages: 264 numpy and scipy (Jones et al, 2001) and stripy (Moresi and Mather, available via 265 https://pypi.org/project/stripy). The example notebooks use the python cartopy package to 266 generate maps (Met. Office, 2010). The models are provided in a python package

267 miller_alaskamoho_srl2018 available online at

268 (https://pypi.org/project/miller alaskamoho srl2018/) and described in the electronic supplement 269 to this article.

\section{Acknowledgements}

271 This work is supported by the Australian Research Council Discovery Program DP150102887.

272 Many thanks are due to S.M. Roeske and A. Till for inspiring discussions and encouragement. 
Allam, A.A., V. Schulte-Pelkum, Y. Ben-Zion, C. Tape, N. Ruppert, Z. Ross (2017). Ten Kilometer

276 Vertical Moho Offset and Shallow Velocity Contrast Along the Denali Fault from Double-

277 difference Tomography, Receiver Functions, and Fault Zone Head Waves, Tectonophysics. $278 \quad 72156-69$.

279 Bassin, C., G. Laske, and G. Masters (2000). The Current Limits of Resolution for Surface Wave 280 Tomography in North America. EOS Transactions, 81, F897.

281 Brennan, P.R., H. Gilbert, and K.D. Ridgway (2011). Crustal structure across the central Alaska 282 Range: Anatomy of a Mesozoic collisional zone: Geochem., Geophys., Geosys. 12, 283 doi:10.1029/2011GC003519.

284 Crotwell, H. P., and T. J. Owens (2005), Automated receiver function processing, Seism. Res. Lett., $285 \quad \underline{76,702-708}$

286 Eagar, K.C., and M.J. Fouch (2012). FuncLab: A MATLAB Interactive Toolbox for Handling 287 Receiver Function Datasets, Seismo. Res. Lett. 83 596-603, doi:10.1785/gssrl.83.3.596.

288 Gilbert, H. (2012). Crustal structure and signatures of recent tectonism as influenced by ancient 289 terranes in the western United States. Geosphere. 8(1) 141-157. doi: 10.1130/ges00720.1

290 IRIS DMC (2010), Data Services Products: EARS EarthScope Automated Receiver 291 Survey, https://doi.org/10.17611/DP/EARS.1.

292 Jones E, Oliphant E, Peterson P, et al. SciPy: Open Source Scientific Tools for Python, 2001-, 293 http://www.scipy.org/ [Online; accessed 2018-07-26].

294 Kennett, B.L.N., E.R. Engdahl, and R. Buland (1995). Constraints on seismic velocities in the 295 Earth from travel times, Geophys. J. Int. 122 108-124.

296 Levander, A., and M.S. Miller (2012). Evolutionary aspects of the lithosphere discontinuity 297 structure in the Western U.S. Geochemistry Geophysics Geosystems. 13, doi: 298 doi:10.1029/2012GC004056. 
Meltzer, A., R. Rudnick, P. Zeitler, A. Levander, G. Humphreys, K. Karlstrom, E. Ekstrom, C.

300 Carlson, M. Dixon, M. Gurnis, and P. Shearer (1999). The USArray initiative. Geological Society 301 of America TODAY. 9 8-10.

302 Met Office (2010). Cartopy: a cartographic python library with a matplotlib interface, Exeter, 303 Devon.

304 Miller, M.S., L.J. O'Driscoll, R.W. Porritt, and S.M. Roeske (2018). Multiscale crustal architecture 305 of Alaska inferred from $P$ receiver functions, Lithosphere. 10 267-278. doi.org/10.1130/L701.1

306 Mooney, W. D., G. Laske, and G. Masters (1998). Crust 5.1: a global crustal model at 5x5 307 degrees. Journal of Geophysical Research. 103 727-747.

308 Pasyanos, M. E., G. Masters, G. Laske, and Z. Ma (2014). LITHO1.0: An updated crust and 309 lithospheric model of the Earth. Journal of Geophysical Research. 119(3) 2153-2173, doi: 310 doi:10.1002/2013JB010626

311 Plafker, G., and H.C. Berg (1994). Overview of the geology and tectonic evolution of Alaska. In 312 G. Plafker \& H. C. Berg (Eds.), The Geology of Alaska: Geological Society of America.

313 Porritt, R. W., and M. S. Miller (2018). Updates to FuncLab, a Matlab based GUI for handling 314 receiver functions, Computers \& Geosciences. 111 260-271, 315 doi.org/10.1016/j.cageo.2017.11.022.

316 Renka, R. J. (1997a). Algorithm 772: STRIPACK: Delaunay triangulation and Voronoi diagram on 317 the surface of a sphere. ACM Trans. Math. Softw. 23(3) 416-434. doi: 10.1145/275323.275329 318 Renka, R. J. (1997b). Algorithm 773: SSRFPACK: interpolation of scattered data on the surface 319 of a sphere with a surface under tension. ACM Trans. Math. Softw. 23(3) 435-442. doi: $320 \quad 10.1145 / 275323.275330$

321 Rosen, J. (2017). Seismic array shifts to Alaska. Science, 358 (22) 10.1126/science.358.6359.22.

322 Tape, C., Plesch, A., Shaw, J. H., \& Gilbert, H. (2012). Estimating a Continuous Moho Surface 323 for the California Unified Velocity Model. Seismological Research Letters, 83(4), 728-735. doi: $324 \quad 10.1785 / 0220110118$ 
325 Trabant, C., Hutko, A.R., Bahavar, M., Karstens, R., Ahern, T., and Aster, R. (2012). Data 326 Products at the IRIS DMC: Stepping Stones for Research and Other Applications: Seismo. Res. 327 Lett., 83, 846-854, doi:10.1785/0220120032.

328 Wessel, P., and W. H. F. Smith (1991). Free software helps map and display data, Eos Trans. $329 \quad$ AGU 72, 441 and 445-446.

330 Wilson, F.H., Hults, C.P., Mull, C.G., and Karl, S.M., comps., (2015). Geologic map of Alaska: 331 U.S. Geological Survey Scientific Investigations Map 3340, 197, 2 sheets, scale 1:584,000, 332 doi.org/10.3133/sim3340. 


\section{List of Figure Captions}

Figure 1. Distribution of broadband seismic stations used in the analysis, which were accessible from the IRIS data management center (www.iris.edu accessed April 2018), shown as inverted white triangles. Active volcanoes are shown with red triangles (Smithsonian Global Volcanism Program). Inset shows the distribution of earthquakes (blue dots) used in the analysis. Blue inverted triangles indicate the location of the stations shown in Supplementary Figure S1.

Figure 2. A) Moho depth estimates at station locations shown as colored dots. Major faults are

342 indicated with thin black and grey lines, dashed where approximately located. B) Moho depth

343 map with simplified litho-types overlain with the faults (Wilson et al., 2015, Miller et al., 2018).

344 Map colors denote broad crustal categories of terranes, based primarily on dominant bedrock 345 geology and isotopic characteristics: yellow_continental and marginal basins, including Yukon

346 Tanana terrane (YTT); orange-red-ocean plateau and arc, including the Wrangellia composite

347 terrane (WCT); green_clastic marine basins; purple-accretionary complex.

Figure 3: $(A)$ Mean errors and (B) rms variation about the mean in the prediction of each data point using information from all other data points and a uniform error assumption to compute the model interpolant. The measures are averages over all model parameters for each data point. (C) 352 is the model score extracted from the measures in $(A, B)$. Fitting the model 2: choice of parameters

354 Figure 4. The mean error $(A)$ from 250 realizations in predicting the test data for $p_{1}, p_{2}$ and the 355 mean error $(B)$ for predicting all data (test and training). The white circle is the minimum value for 356 each case. The triangles lie within $1.25 \%$ of the minimum error. The white squares are the points 357 which lie in the $1.25 \%$ range of both measures. 
360 Figure 5. (a) Moho surface computed from the procedure described above. (b) Slope of the 361 Moho surface in (a). Major faults are indicated with thin black and white lines, dashed where 362 approximately located.

363

\section{Supplementary Figure Caption}

365 Figure S1. Receiver gathers for three stations A) TA.H2OK, B) AK.VRDI, and C) AK.BMR with 366 locations indicated in Figure 1 and in the lower right of each panel. The first large amplitude 367 positive amplitude signal (in red) at depth is interpreted to be the Moho signal, with the depth 368 (in km) indicated in each panel as picked within the Funclab (see Porritt and Miller, 2018) picking 369 routine. The orange line marks $35 \mathrm{~km}$ depth. The number of receiver functions (RF) used to 370 create the gather is listed in the bottom right of each panel. 


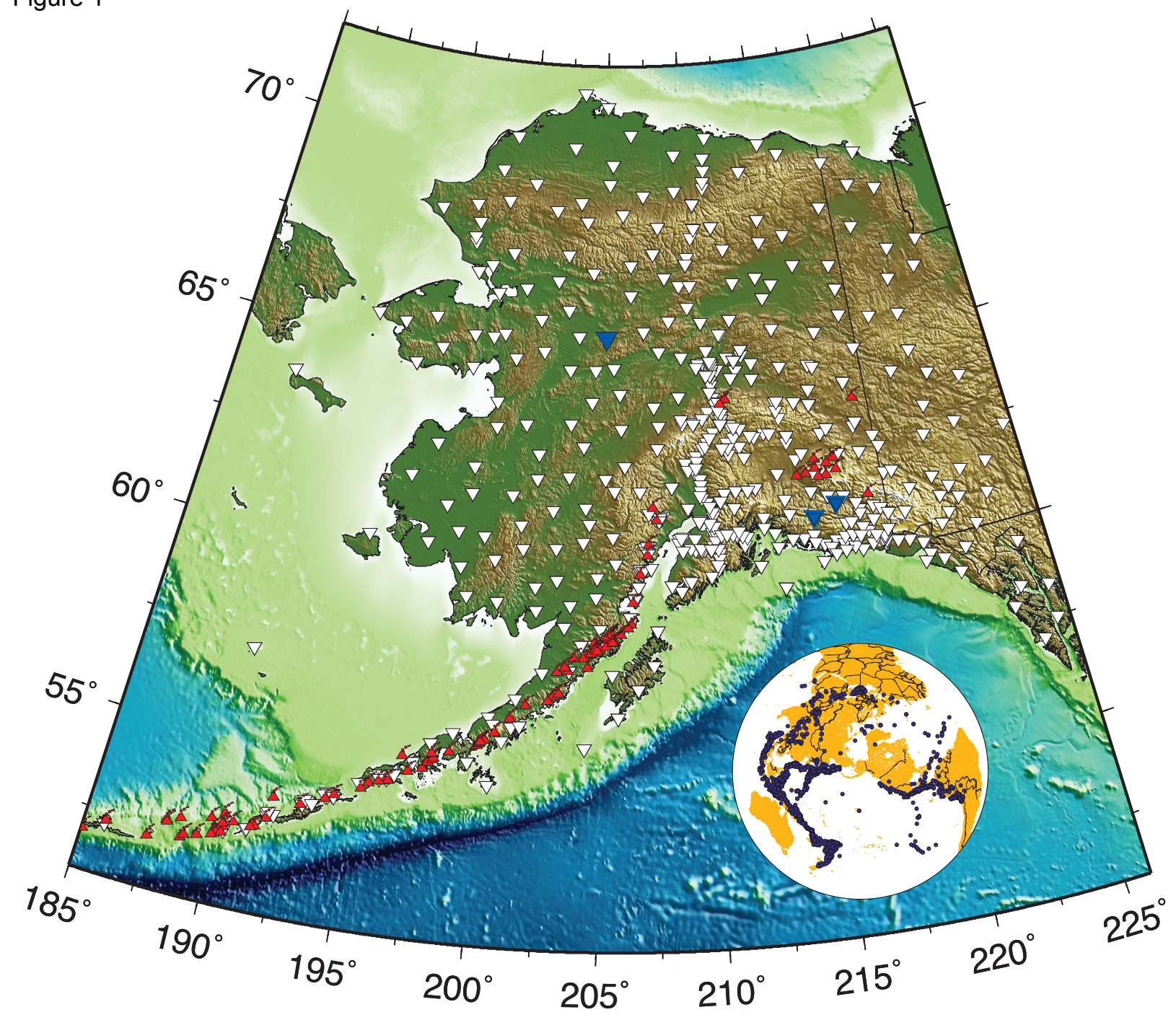

$185^{\circ}$ कोit 
A)

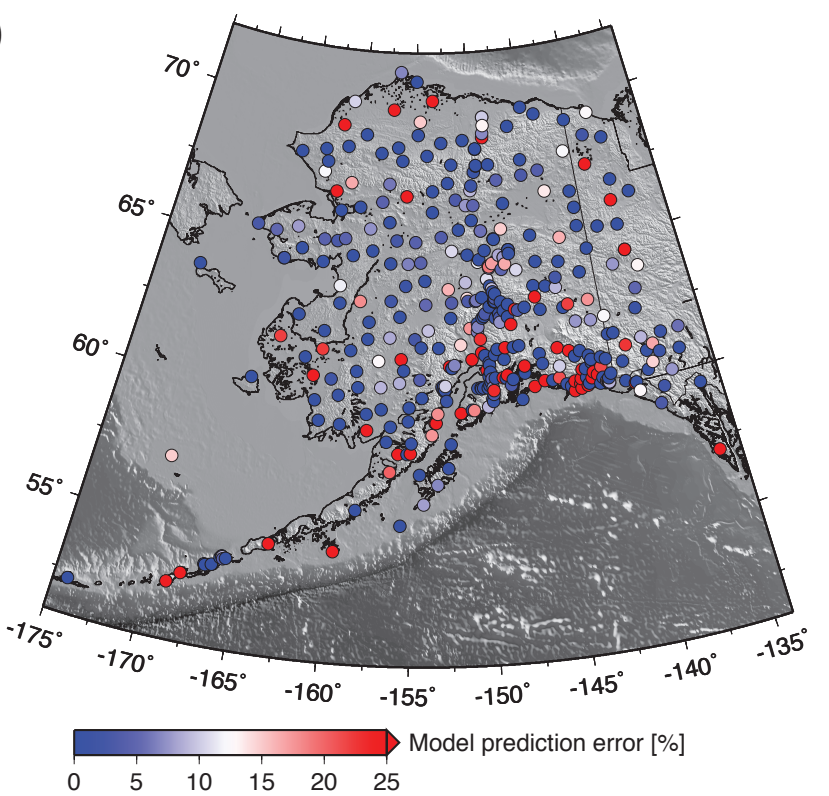

B)

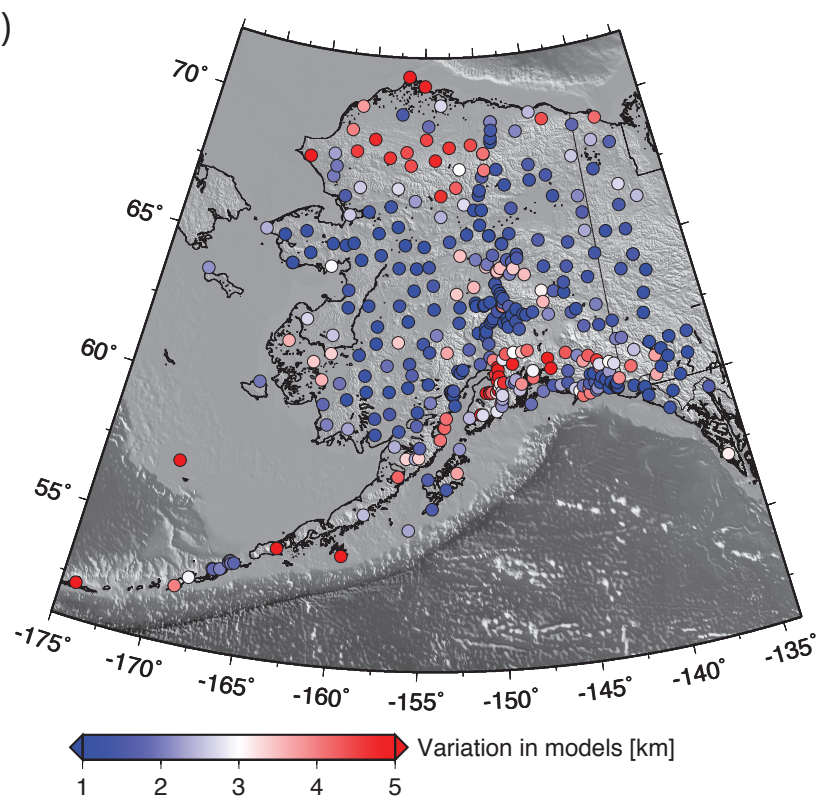

C)

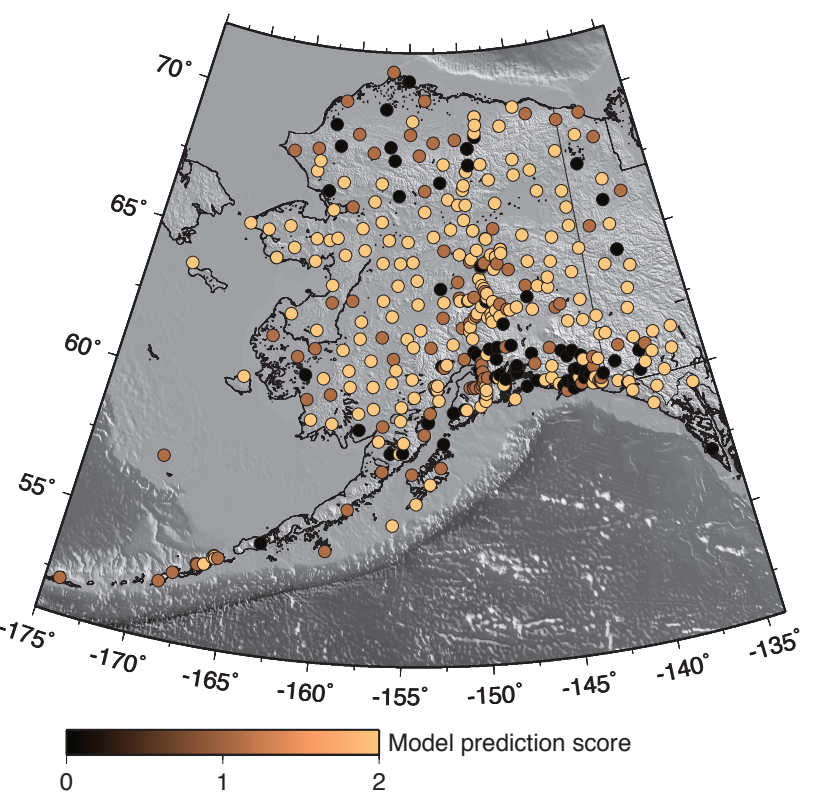



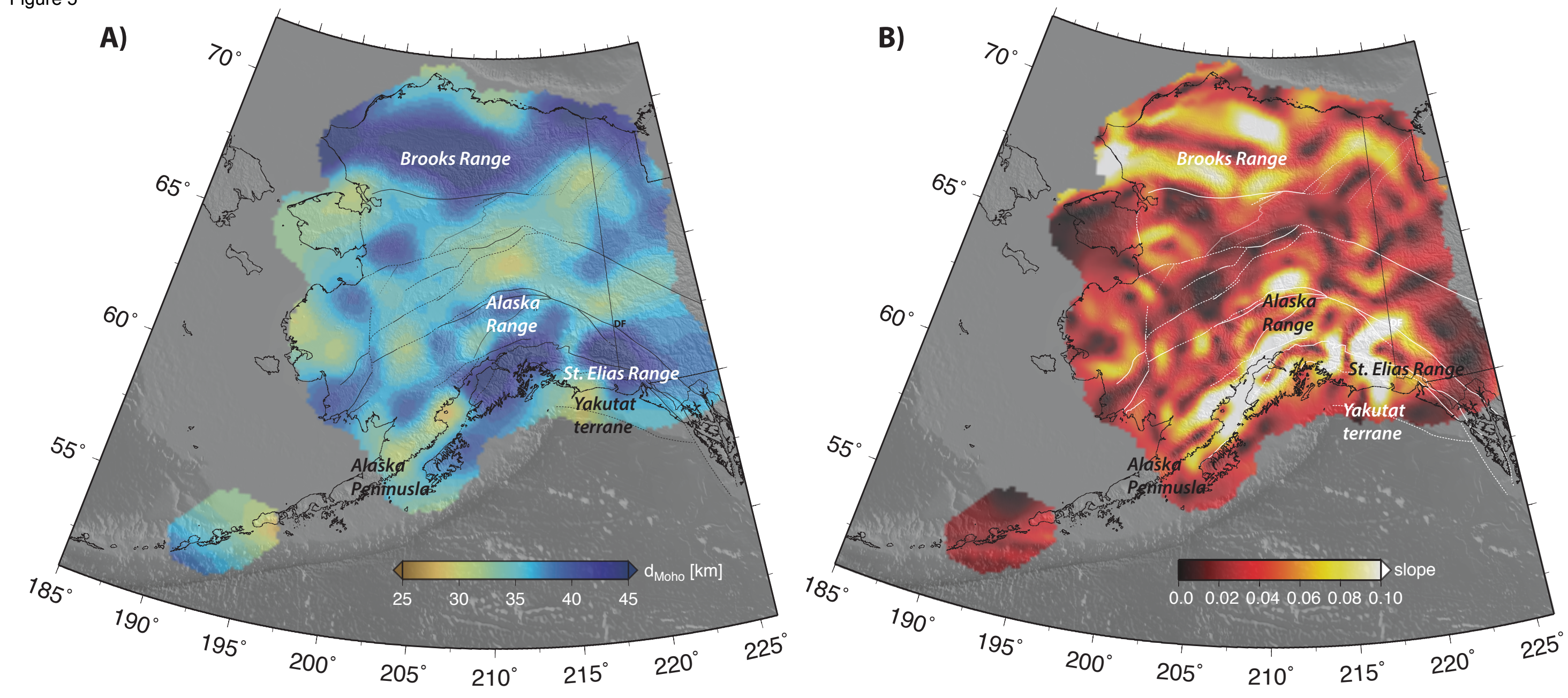



\section{Electronic Supplementary Main Page}

2 The electronic supplementary material includes two tables, which are the DOls for all the

3 networks used in the analysis (Table S1) and the Moho Depth estimates (Table S2). It

4 also includes one figure (Figure S1) that provides texample receiver function stacks at

5 three stations (A) TA.H2OK, B) AK.VRDI, and C) AK.BMR.

6 The electronic supplementary also includes the following instructions for installing the

7 python package to access the model / data and the sample notebooks. Notebooks are

8 also provided for reproducing (or modifying) the interpolating models.

\section{$9 \quad$ Installation instructions}

10 The package can be installed standalone using pip or it can be run through docker without

11 needing specific installation.

12 These notebooks can be viewed with jupyter but will only run if all the software dependencies

13 have been installed (see Installation through pip below.

14 In nearly every instance we recommend using the (self-contained) docker version. We have

15 provided some useful bash shortcuts that make the docker commands easier to remember

16 (see Installation through docker below).

\section{Installation through docker}

18 First it is necessary to install the free docker, community edition from the docker store

19 (https://store.docker.com/search?offering=community\&type=edition) for your platform.

20 \# Download the image with the scripts and data

21 docker pull lmoresi/docker-miller-moho:1.0

22 That's it !

23 To test the installation, try the following 
Command line docker examples

2 This help message

5 Install the bash helpers in the current directory

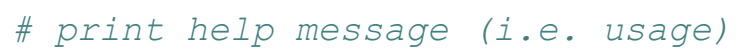

source moho_bash_utils.sh

10 Install the documentation / scripts and notebooks in the current directory

11 \# print help message (i.e. usage)

12 source moho_bash_utils.sh

13 moho-docker-sh install_examples

14 Run a local python script

15 \# run my script.py with python in the docker container

16 source moho_bash_utils.sh

17 moho-docker-sh my_script.py

18

\section{Installation through pip}

20 Note - these instructions are not comprehensive and if they make little or no

21 sense to you, go back up to the docker installation.

22

23 The package requires a python interpreter and the pip package manager

24 (https://packaging.python.org/tutorials/installing-packages — accessed 2018.07.26). The

25 meshing and interpolation package, stripy, requires a fortran compiler to be installed in 
1 a discoverable location on the machine. Other dependencies are listed below and can be

2 installed by whichever package manager(s) you prefer. pip can be used to install this

3 package and the stripy dependency even if the commonly used conda package manager

4 is used to install everything else. On a completely clean install, conda is able to install

5 every required dependency and is therefore recommended

6 (https://conda.io/docs/index.html — accessed 2018.07.26)

7

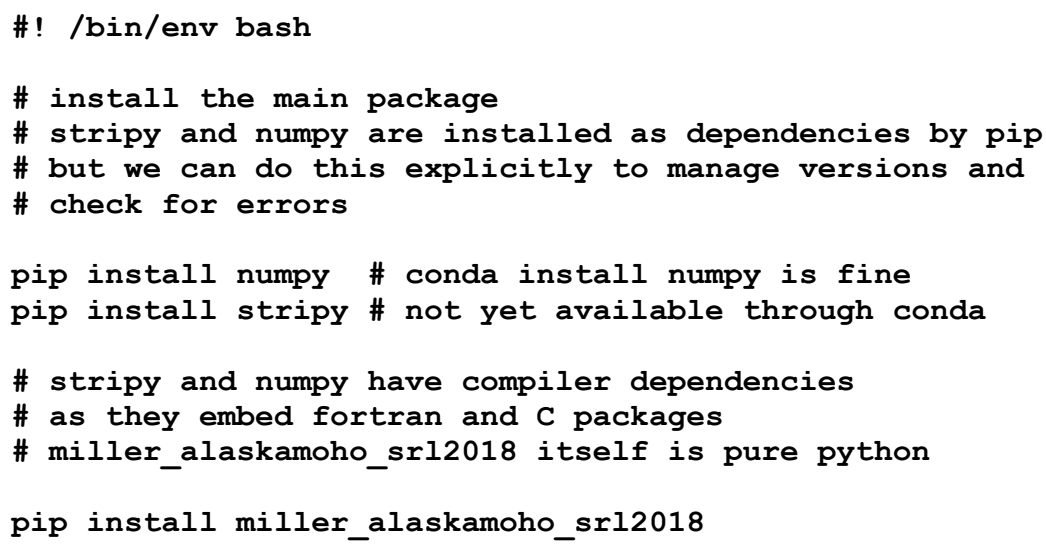

The following script tests the installation. It also demonstrates how read the data array and how to use the interpolation method of the surface_model class.

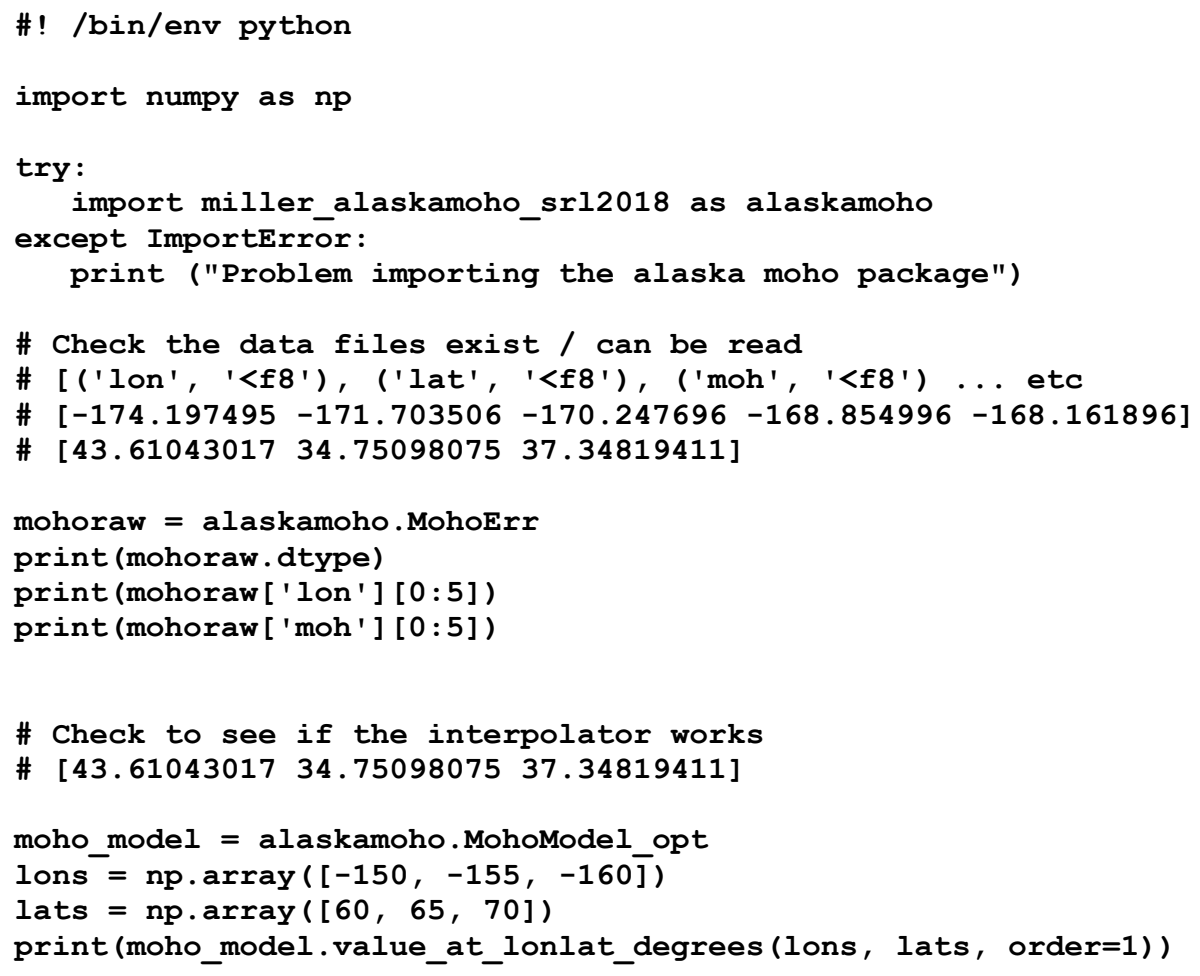


\# install documentation in user-specified location

\# Should install in the current directory as AlaskaMohoExamples

alaskamoho.documentation.install_documentation()

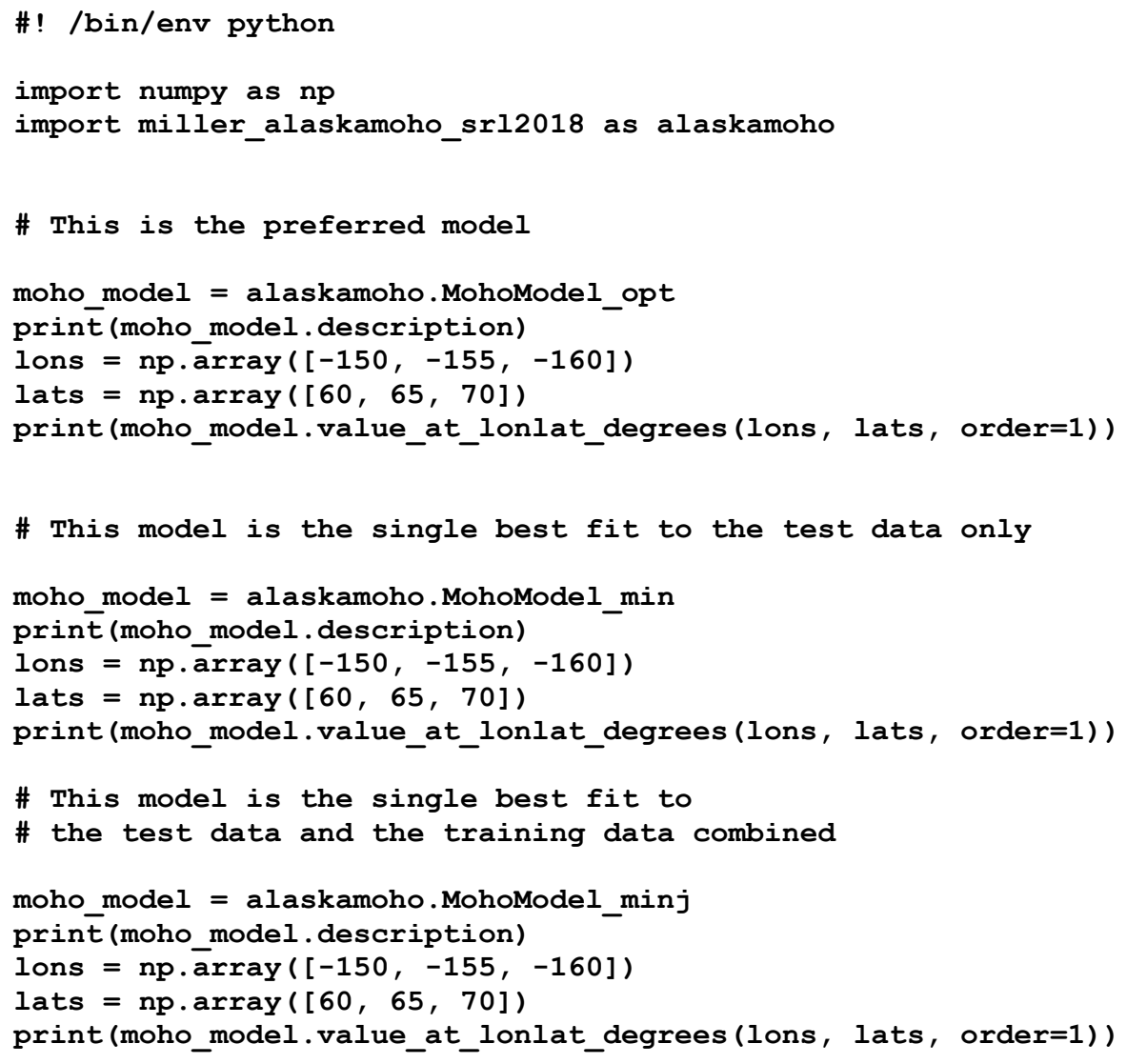

The usage of the package may be self evident at this point, but further examples including samples of the maps and plots in the body of the paper are provided in the jupyter notebooks.

\section{Jupyter Notebooks}

The jupyter notebook examples are self-documented, runnable code to reproduce specific figures in the paper. We also provide the notebooks that we used to build all of the models. The following script installs the necessary dependencies to run all of the notebooks

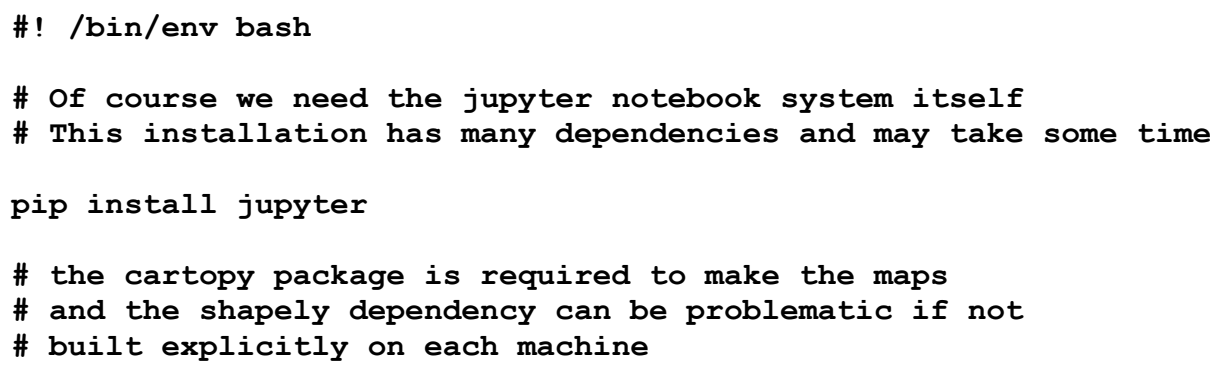




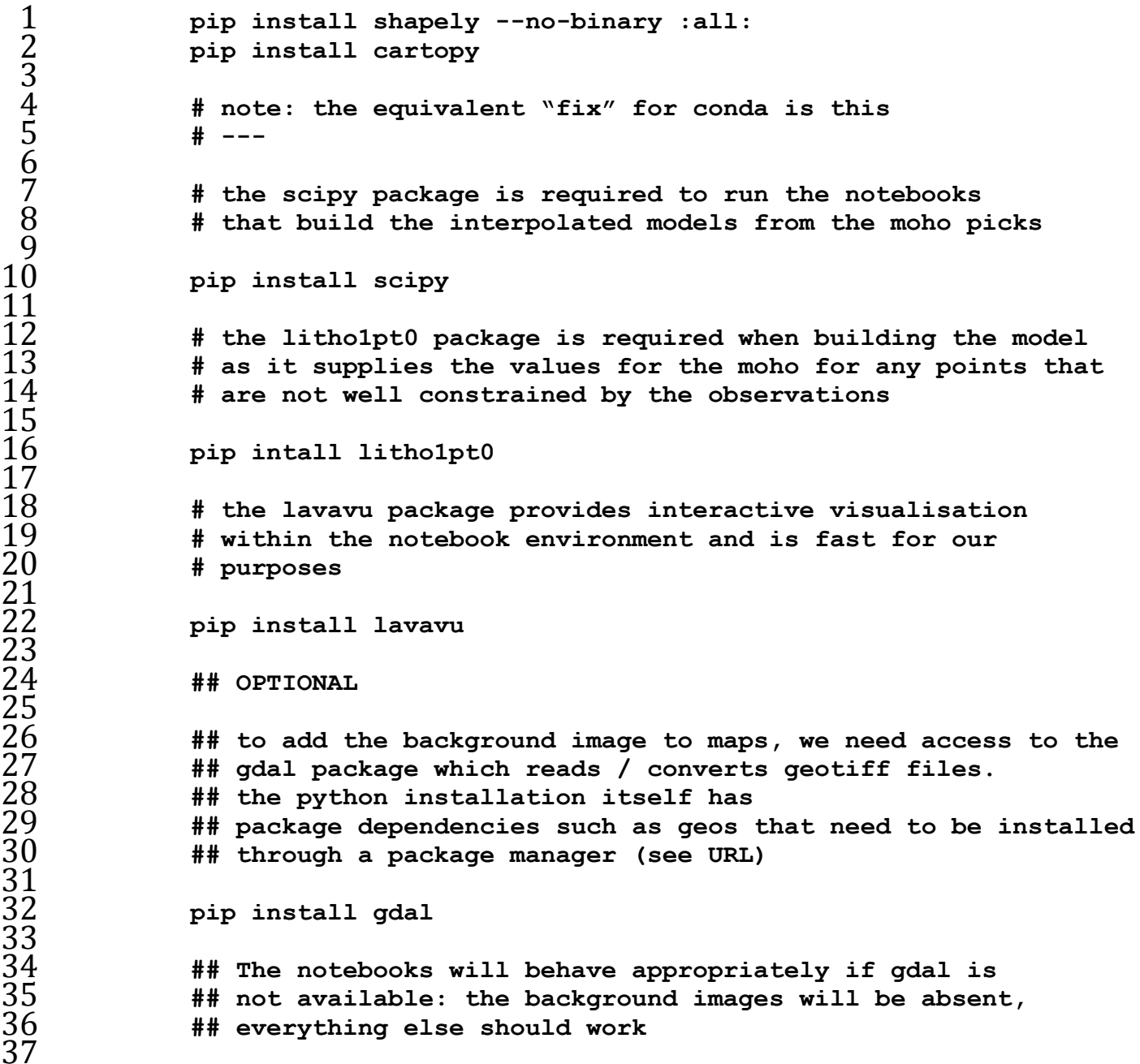

39 The installation of the example notebooks is done through the 40 miller_alaskamoho_srl2018 package itself. The following script will install the 41 documentation in the path you choose (or in the current working directory by default)

\#! /bin/env python

import miller_alaskamoho_srl2018 as alaskamoho

\# install documentation in user-specified location

\# change the path to suit or leave blank to install

\# in the current directory under AlaskaMohoExamples

alaskamoho.documentation.install_documentation (path="path/to/notebooks")

The structure of the examples directory installed from the package is as follows:

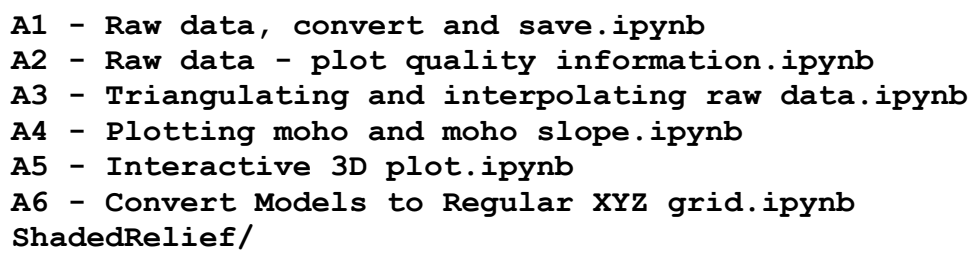




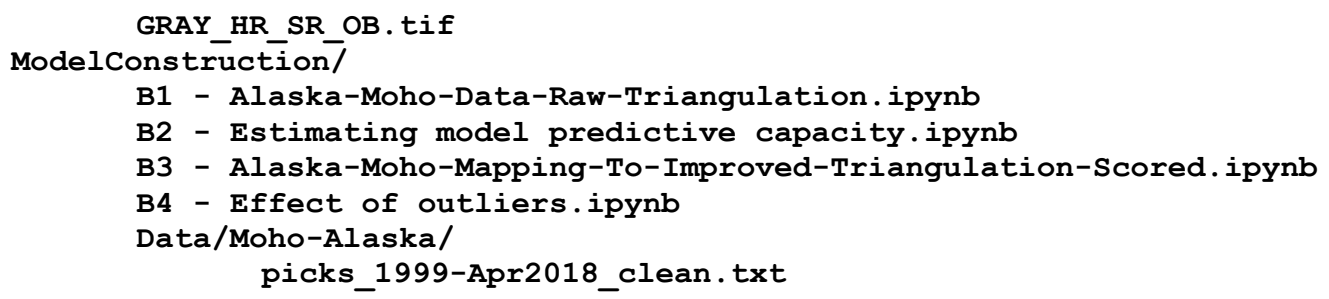

The jupyter notebook system should be started from the location where the examples were installed. Providing a notebook name at startup bypasses the notebook browser.

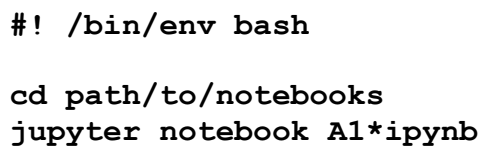

A brief description of the notebooks that are provided is given in the table below.

\section{A: Using the models \\ Notebook - description}

A1 - Raw data, convert and save.ipynb - python functions to read the model data files and convert to text or csv files for use elsewhere.

A2 - Raw data - plot quality information.ipynb - the model takes the form of the raw data plus various measures to indicate the ability of the model to predict data around each data point. These notebooks reproduce the information in Figure 3.

A3 - Triangulating and interpolating raw data.ipynb - this notebook demonstrates how to generate a spherical triangulation of the moho depth data and interpolate to any point within the triangulation. The irregularity of the points means that no smoothing or gradient information can be obtained.

A4 - Plotting moho and moho slope.ipynb - this notebook reads the processed surface models on a fine, triangulated grid and demonstrates how to interpolate the information within that triangulation to plot or obtain a cross section.

A5 - Interactive 3D plot.ipynb - using the lavavu interactive viewer in the notebooks to compare the smooth moho surface with the original data points 
A6 - Convert Models to Regular XYZ grid - interpolate the data and the quality measures to a 'regular' grid in longitude / latitude coordinates for plotting

2 The notebooks that can be used to reproduce the data scoring and model fitting are

3 listed below. These assume some familiarity with the details of the python dependencies

4 such as stripy, scipy and numpy.

\section{B: Building the models}

Notebook - description

B1 - Alaska-Moho-Data-Raw-Triangulation.ipynb - python functions to read the original picks, verify uniqueness of locations for the purposes of triangulation, triangulate, interpolate and plot.

B2 - Estimating model predictive capacity.ipynb - construct the smooth model with parameters for resolution and smoothness, perform multiple realisations with training I testing subsets of the data, score models according to predictive capability of smooth models for each data point.

B3 - Alaska-Moho-Mapping-To-Improved-Triangulation-Scored.ipynb - use the scoring from (B2) as the weights for least squares fit for all model smoothness and resolutions and determine preferred models.

B4 - Effect of outliers.ipynb - the process in (B2) identifies some clear outliers. We re-ran the model fit excluding the shallow and deep outliers to understand where the complex moho does not produce a clear single-surface. This information is provided as contour maps and a cross section. 


\section{TA.H20K - North-central Alaska}

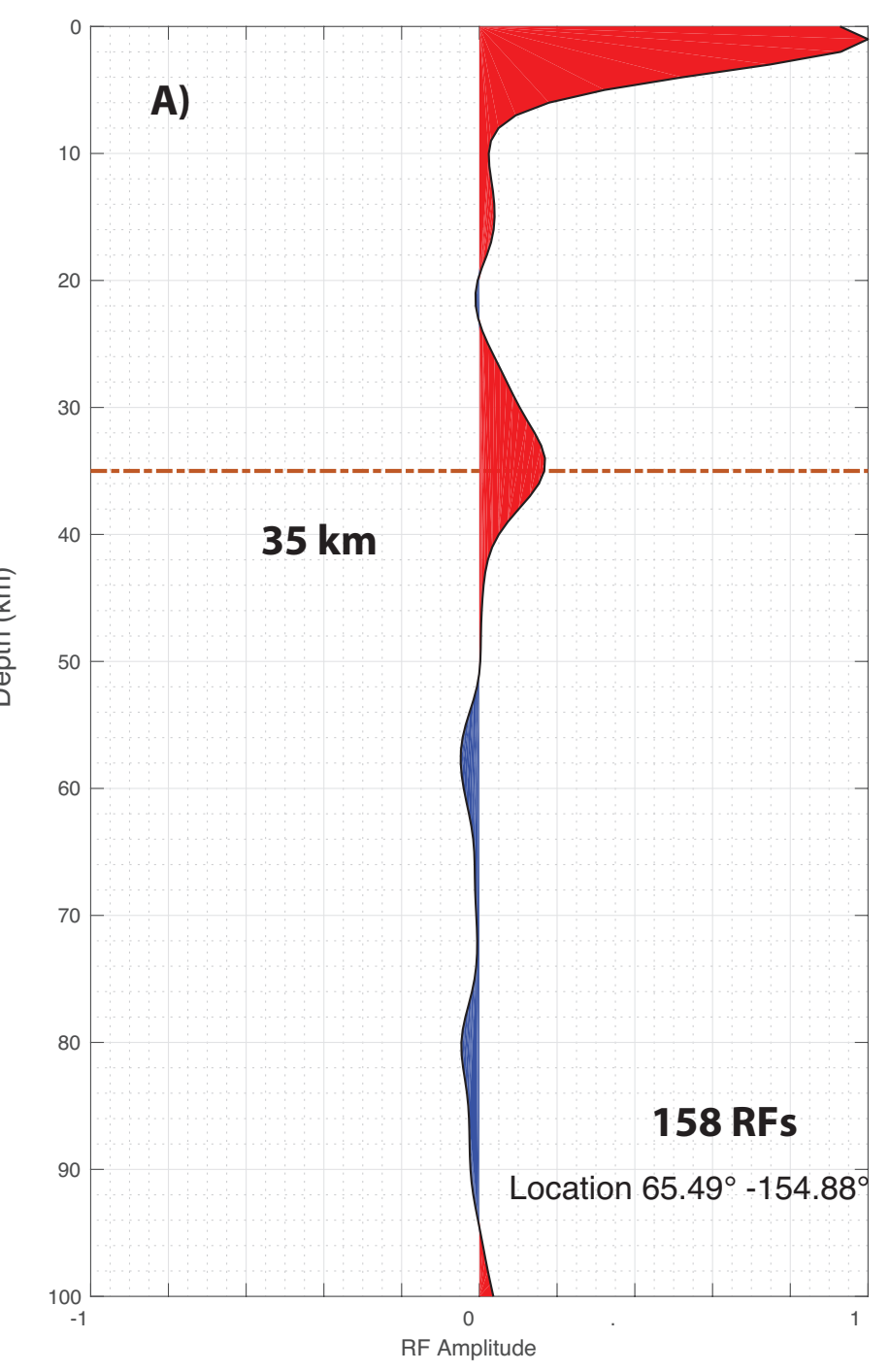

AK.VRDI - Accretionary Complex

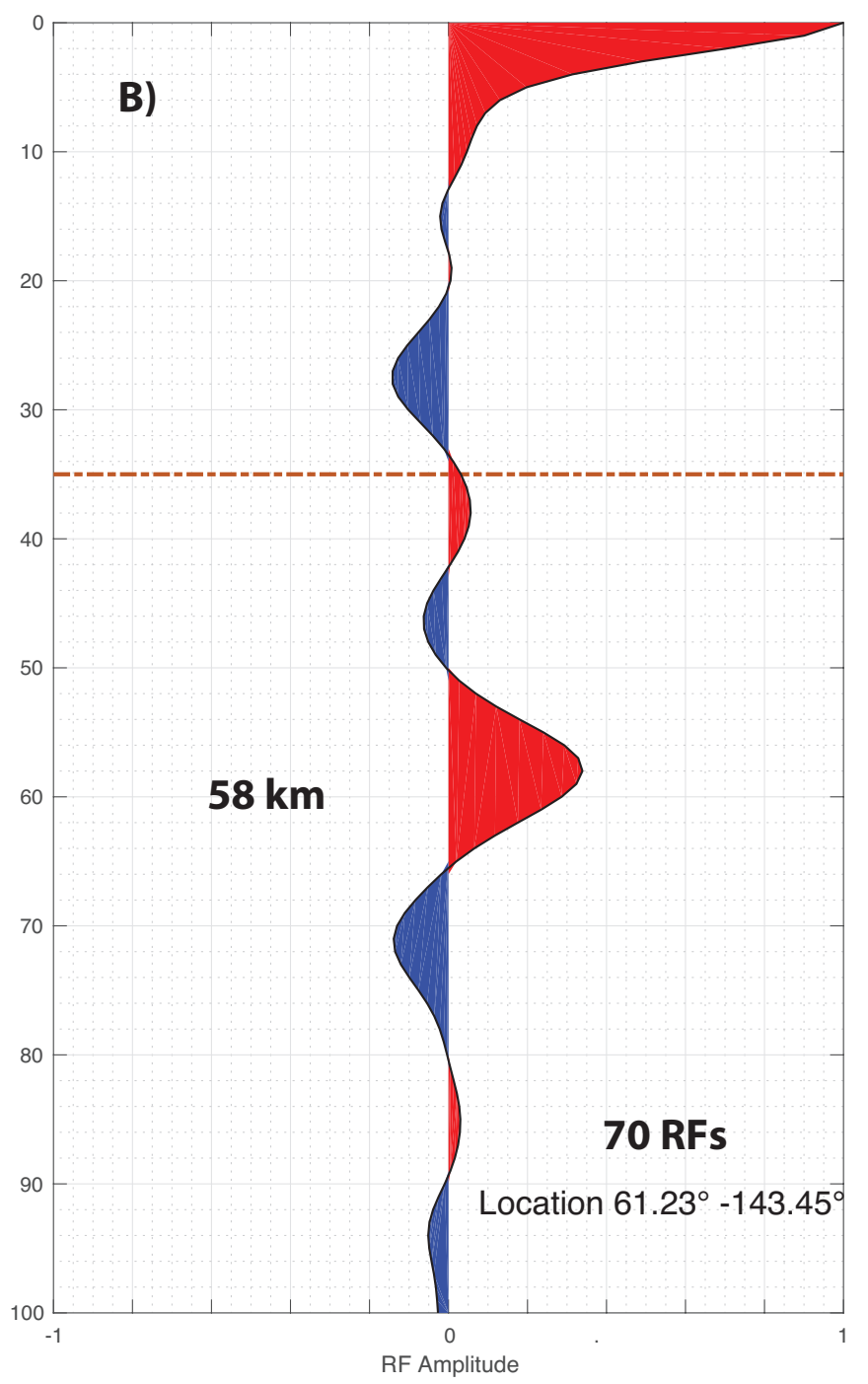

AK.BMR - Accretionary Complex

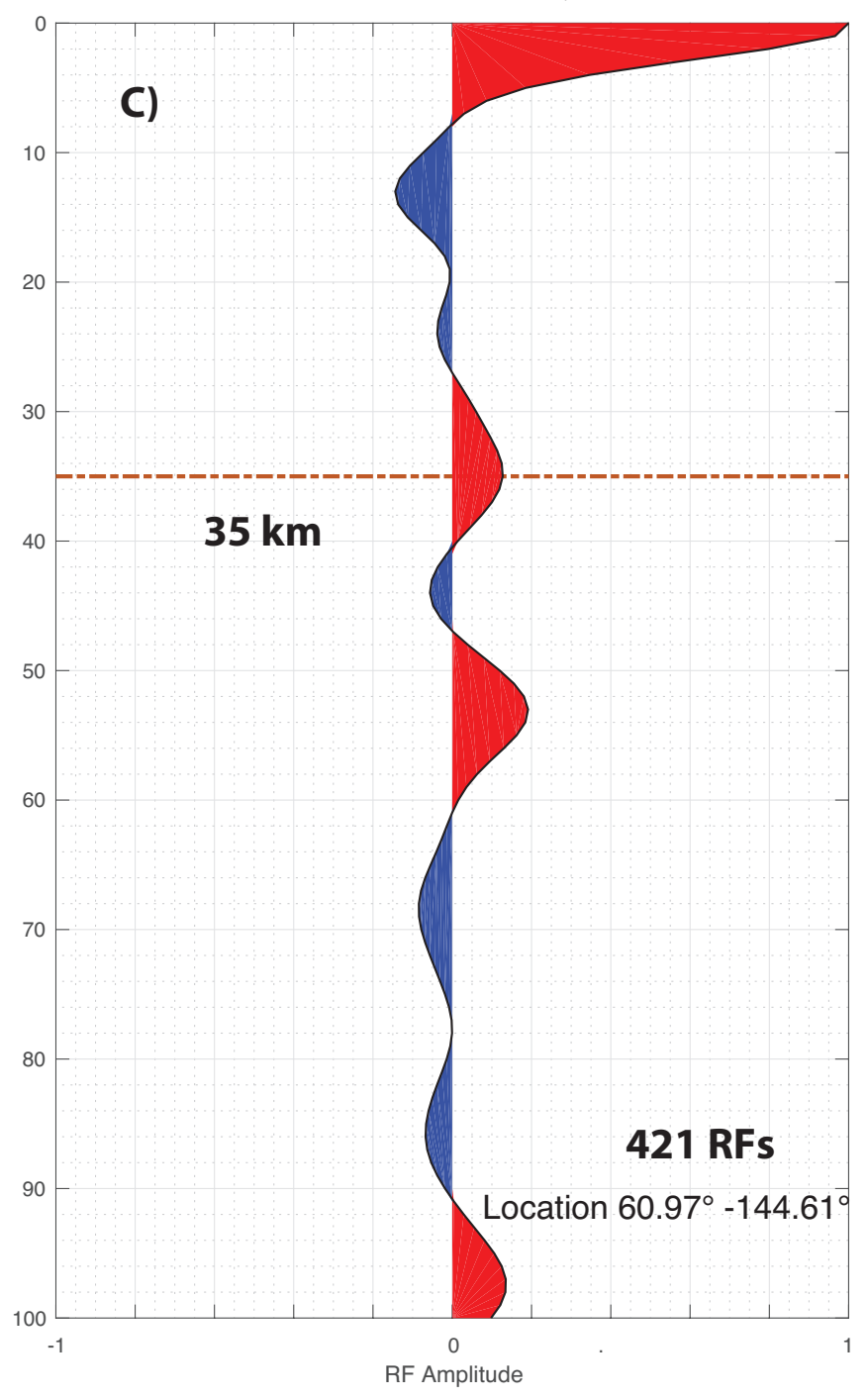

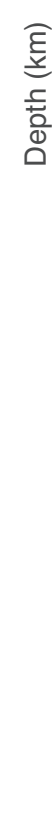

